\title{
Capacity Improvement through Buffer-Aided Successive
}

Opportunistic Relaying

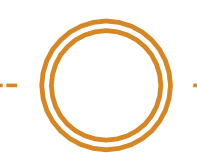

N. NOMIKOS ${ }^{1}$, D. VOUYIOUKAS ${ }^{1}$, T . CHARA LAMB OUS ${ }^{2}$, I KRIKI DIS ${ }^{3}$, D . N . SKOUTAS ${ }^{1}$, AND M. JOHANSSON ${ }^{2}$
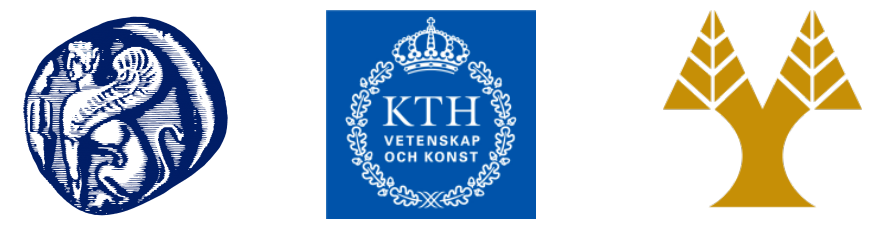

1 UNIVERSITY OF THE AEGEAN, DPT. OF INFORMATION AND COMMUNICATION SYSTEMS ENGINEERING, KARLOVASSI, GREECE

2 ROYAL INSTITUTE OF TECHNOLOGY (KTH), DPT. OF ELECTRICAL ENGINEERING AND ACCESS LINNAEUS CENTER, STOCKHOLM, SWEDEN

3 UNIVERSITY OF CYPRUS, DPT. OF ELECTRICAL AND COMPUTER ENGINEERING, NICOSIA, CYPRUS 


\section{Contents}

- Introduction-Motivation

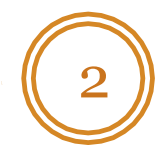

○ Cooperative relaying

○ Buffer-aided relaying

○ Opportunistic relaying

- Successive relaying

- System model

- Buffer-aided successive opportunistic relaying

- Contributions

- Relay-pair selection algorithm

- Numerical results

- Outage probability

$\circ$ Average capacity

$\circ$ Average delay

- Conclusions

- Future directions 


\section{Introduction-Motivation}

- The development of spectral efficient protocols is an important research area

o The goal of providing data at Gbps levels requires the efficient use of spectral, temporal and spatial resources

- Multi-hop communications is another field which has attracted the attention of the academia and the industry

o Cooperative relaying employs intermediate nodes to relay the information towards the destination 


\section{Cooperative relaying}

- Cooperative relaying is a major element of next generation wireless networks

- Relaying increases the diversity gain by offering alternative and independent transmission paths [Laneman et al., 2004]

- Enhancing the relays with buffering capabilities increases the degrees of freedom in the network

- Improved diversity as relays which have packets in their buffers can be scheduled to transmit to the destination

- Opportunistic relay selection improves the usage of network resources by selecting one best relay among multiple relay nodes [Bletsas et al., 2006]

○ The best relay is selected based on the end-to-end channel quality without sacrificing the outage performance

- Successive relaying [Ding et al., 2012] allows the source and the relay to transmit simultaneously

○ Full-duplex operation but with inter-relay interference (IRI) 


\section{Opportunistic relaying}

(5)

- Opportunistic relay selection is based on the max-min criterion and its variations [Bletsas et al., 2006], [Michalopoulos et al., 2008], [Krikidis et al., 2009]

$\circ$ In these works the source and the relay transmitted in orthogonal time-slot, thus reducing the end-to-end rate by one-half

- In opportunistic relay transmission only one relay is activated after a selection process

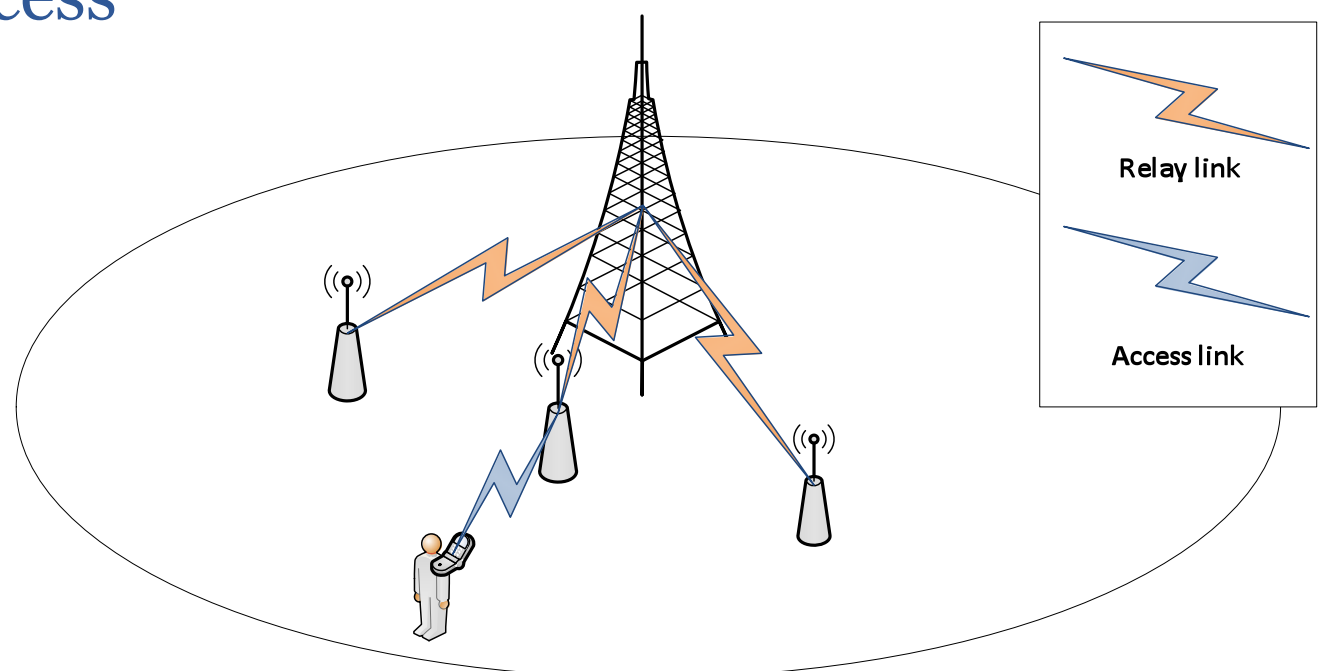




\section{Successive relaying}

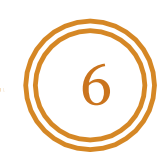

- Successive relaying is coupled with relay selection to combine the advantages of these techniques

o In successive relaying we have two overlapping transmissions

o In [Nomikos et al., 2012/1] the IRI was canceled at the relays for cases of strong interference

o In [Nomikos et al., 2012/2] relays with multiple interfaces are employed and out-of-band transmissions are possible

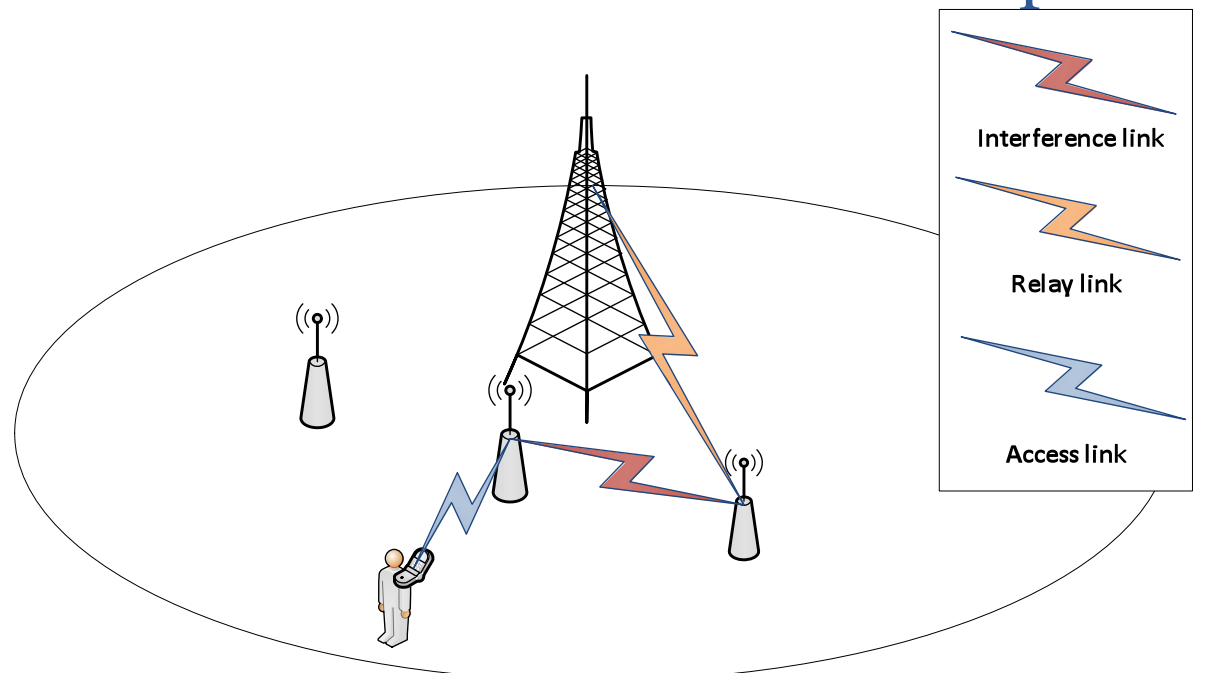




\section{Buffer-aided relaying}

- The addition of buffering at the relays has been suggested as a way to further improve the diversity of the network

- Buffering decouples the receiving relay at the previous time slot to be the transmitting relay at the next slot

○ In [Ikhlef et al., 2012/1] max-max relay selection (MMRS) was proposed in which the relay with the best source-relay $(S R)$ link is selected for reception and the relay with the best relay-destination $(R D)$ link is selected for transmission

O Also, in [Ikhlef et al., 2012/1] hybrid relay selection (HRS) is presented which combines the max-min with max-max relay strategies when buffers are almost full to avoid diversity losses

O In [Krikidis et al., 2012] max-link selection was proposed where the best link among the $S R$ and $R D$ is selected in each time-slot

- In [Ikhlef et al., 2012/2] MMRS is coupled with successive relaying leading to the space full-duplex MMRS (SFD-MMRS)

- No IRI is considered as relays are isolated 


\section{System model}

(8)

- A simple cooperative network consisting of one source $S$, one destination $D$ and a cluster $C$ with $K$ decode-and-forward (DF)

- Rayleigh block fading

- Half-duplex buffer-aided relays

- IRI arises between the selected relay-pair

- Global CSI is available

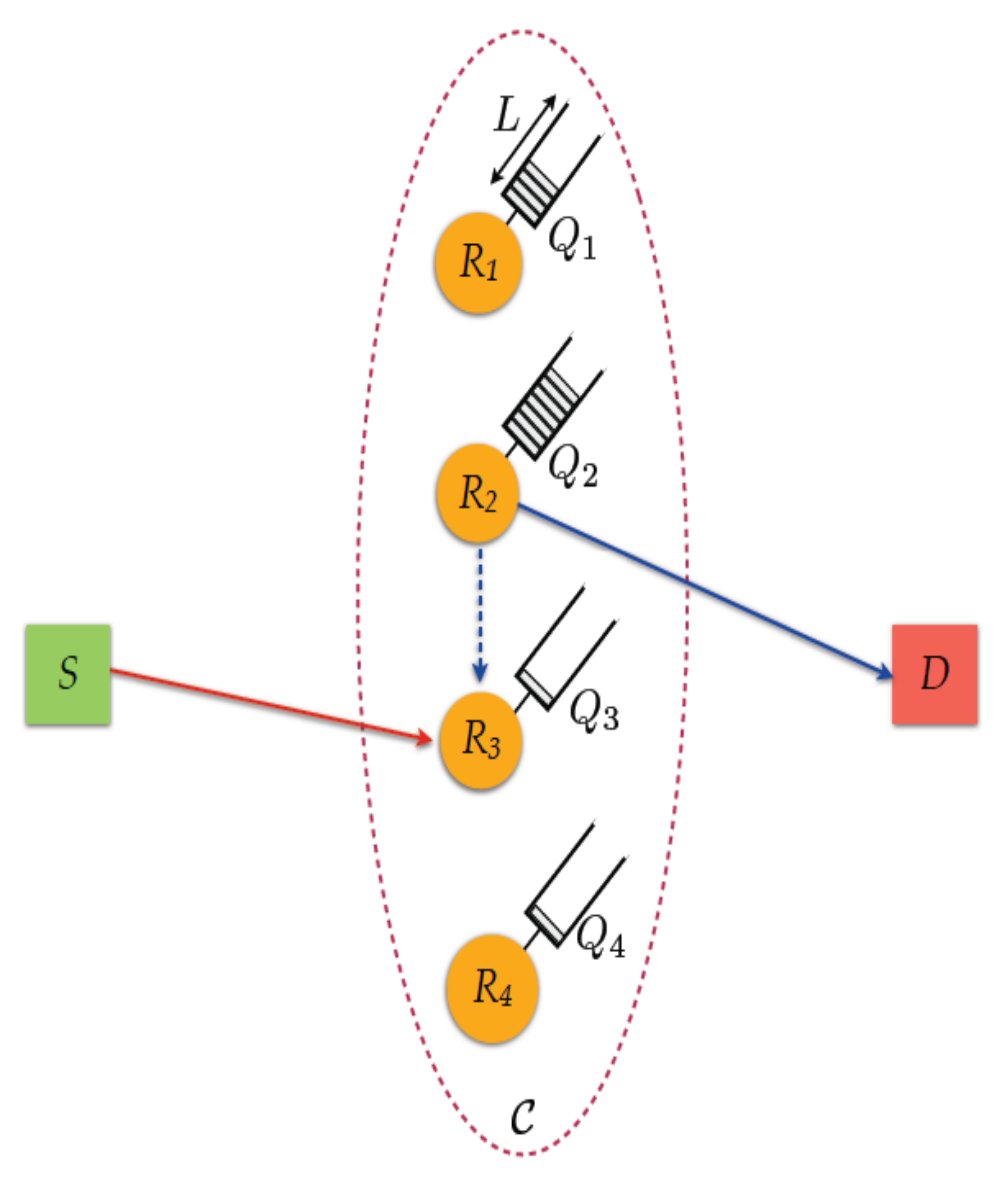




\section{Buffer-aided successive opportunistic relaying}

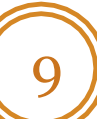

- In this work a buffer-aided successive opportunistic relaying (BA-SOR) scheme is proposed

- Buffering at the relays is combined with opportunistic and successive relaying

o Opportunistic relaying and buffering increase the chances of improved diversity

o Successive relaying aims to leverage the half-duplex loss of relaying

o IRI is introduced in the network requiring efficient mitigation techniques 


\section{Contributions}

(10)

- The selection of the relay-pair that achieves the best end-to-end capacity

- IRI is considered and can be treated either through interference cancellation (IC) or through interference avoidance (IA)

$\circ$ If the inter-relay link is strong, the receiving relay can decode the interfering signal $\rightarrow$ IC is performed

$\circ$ Otherwise, IA is achieved through the selection of the relaypair with the minimum IRI 


\section{Relay-pair selection algorithm}

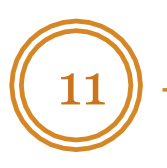

- Due to the concurrent transmissions, relay selection does not depend solely on the quality of the $S R$ and $R D$ links

- IRI is the defining factor in this relay selection policy

- IC is feasible if the inter-relay channel capacity is above the rate threshold $r_{o}$

$$
r_{R_{t} R_{r}} \triangleq \log _{2}\left(1+\frac{g_{R_{t} R_{r}} P_{R_{t}}}{g_{S R_{r}} P_{S}+n}\right) \geq r_{0}
$$

where $g_{i j}$ denotes the channel power between nodes $i, j, P_{i}$ is the transmission power of node $i$ and $n$ is the AWGN

- $K(K-1)$ relay pairs are examined depending on the end-to-end rate they can achieve

- The complexity of the proposed relay selection policy is equal to $O\left(K^{2}\right)$ 


\section{Relay-pair selection algorithm}

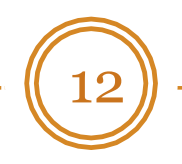

- First, the candidate relay for transmission $t$ is examined

- Then, the IC feasibility check is performed with the other $K-1$ relays

- The best pair is selected according to

$$
\max _{t \in \mathcal{T}}\left\{\min \left[\max _{i \in \mathcal{C}-\{t\}}\left(\frac{g_{S R_{i}}}{g_{R_{t} R_{i}}\left(1-\mathbb{I}\left(R_{t} R_{i}\right)\right)+\mathbb{I}\left(R_{t} R_{i}\right)}\right), g_{R_{t} D}\right]\right\}
$$

where $\mathbf{I}\left(\mathrm{R}_{t} \mathrm{R}_{\mathrm{i}}\right)$ is an indicating factor which is:

$\bigcirc 1$, if IC is feasible

$\circ \mathrm{O}$, if it is not 


\section{Numerical results}

- Comparisons with successive and non successive relay selection schemes

- The successive category includes:

o the SFD-MMRS of [Ikhlef et al., 2012/2] which is considered as the upper bound to our work due to the absence of both IRI and constraints due to full or empty buffers

- The non-successive category includes:

o the HRS of [Ikhlef et al., 2012/1]

○ the max-link of [Krikidis et al., 2012]

- The capacity threshold is $\mathrm{r}_{\mathrm{o}}=2 \mathrm{bps} / \mathrm{Hz}$

- The results are obtained in terms of:

○ outage probability

$\circ$ average end-to-end capacity

$\circ$ average delay 


\section{Outage probability}

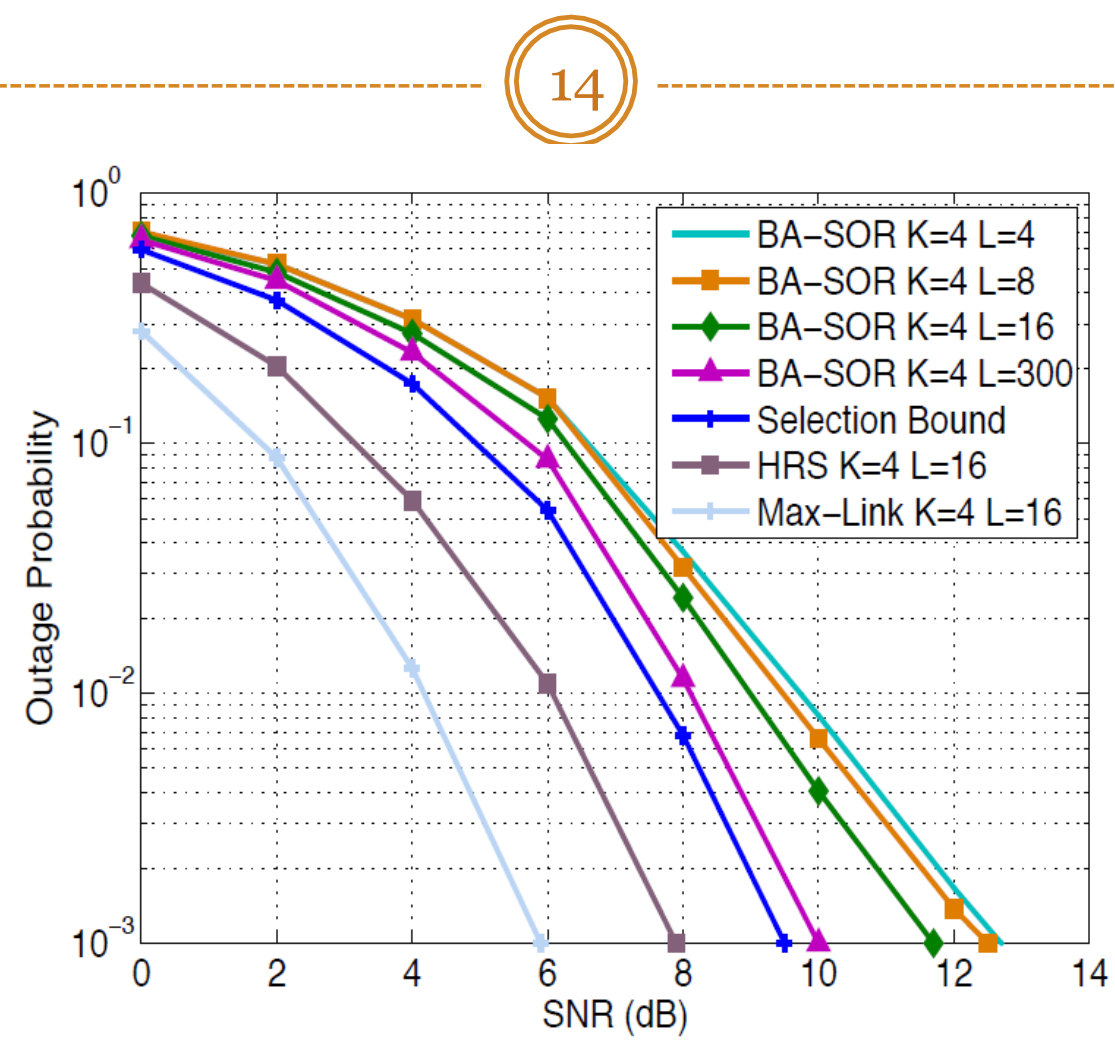

- max-link has the best performance as diversity is equal to $2 \mathrm{~K}$ (high SNR)

- HRS achieves a diversity order equal to K (high SNR)

- SFD-MMRS (Selection Bound) requires two links not to be in outage to achieve successive transmissions, thus leading to increased outage probability

- BA-SOR reduces the gap with the Selection Bound for increasing buffer size $L$ 


\section{Average capacity}

\section{(15)}

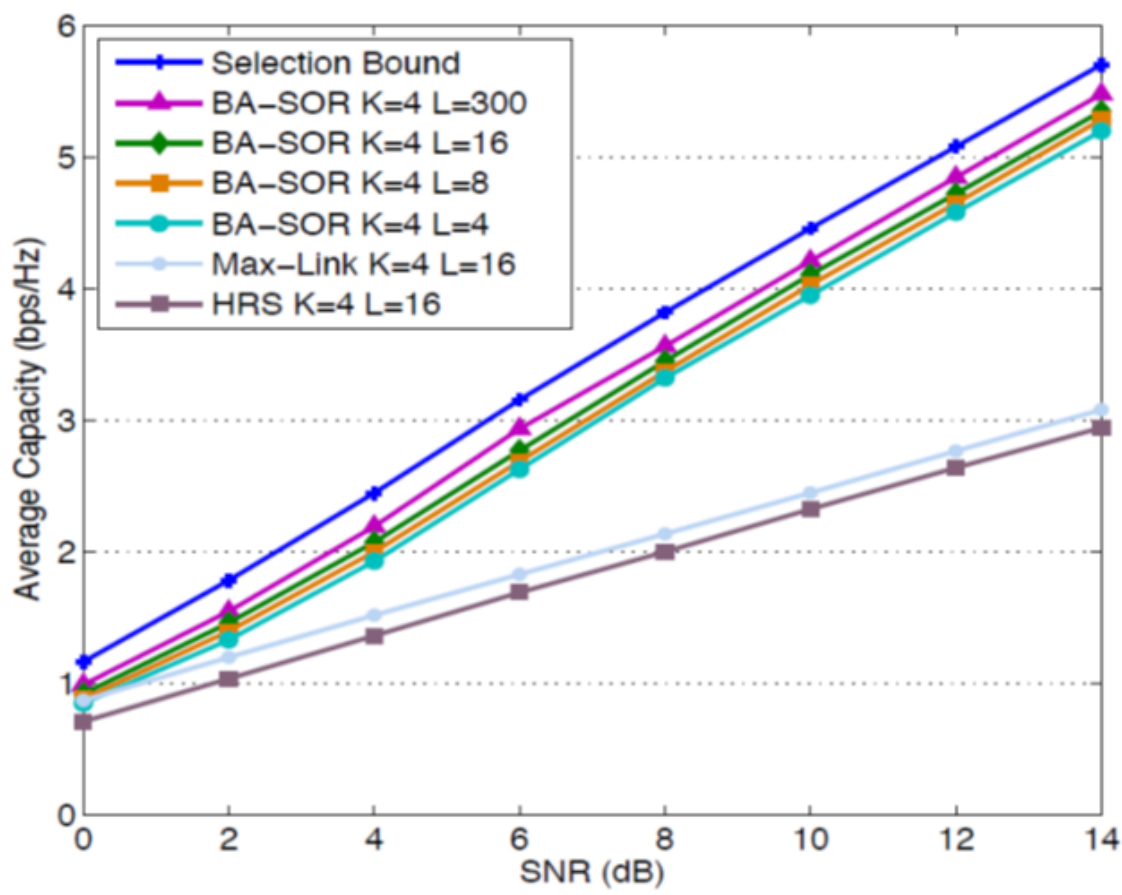

- Clear advantage of the full-duplex schemes which employ two simultaneous transmissions during the whole time-slot

- For high transmit SNR, the capacity of the successive schemes is almost twice the capacity of the half-duplex schemes

- Increased $L$ does not offer big gains in capacity indicating that this metric depends mostly on the number of relays 


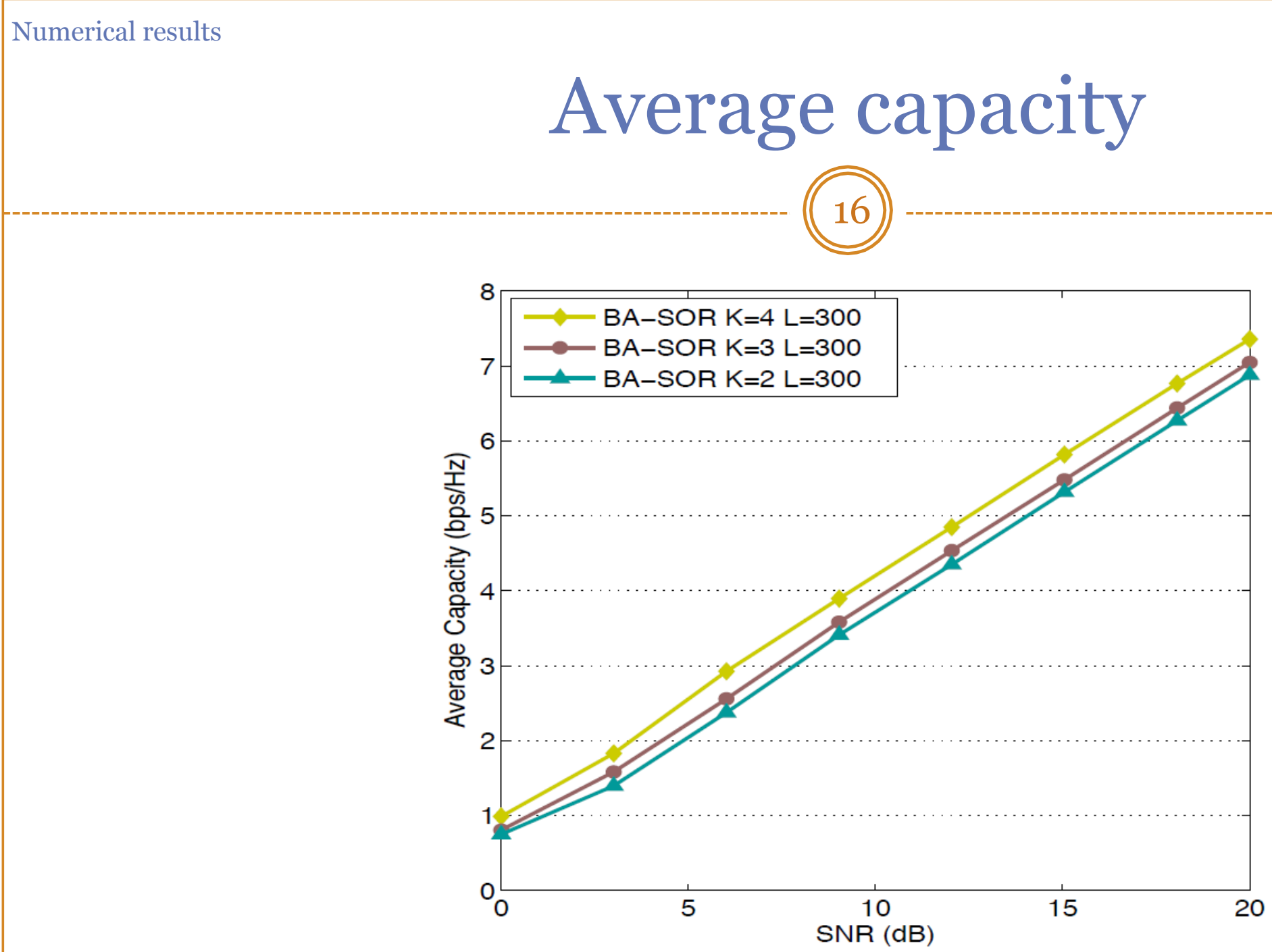

- As the numbers of relays increase $\rightarrow$ the average capacity increases

- Performance gap increases with each relay addition 


\section{Average delay}

(17)

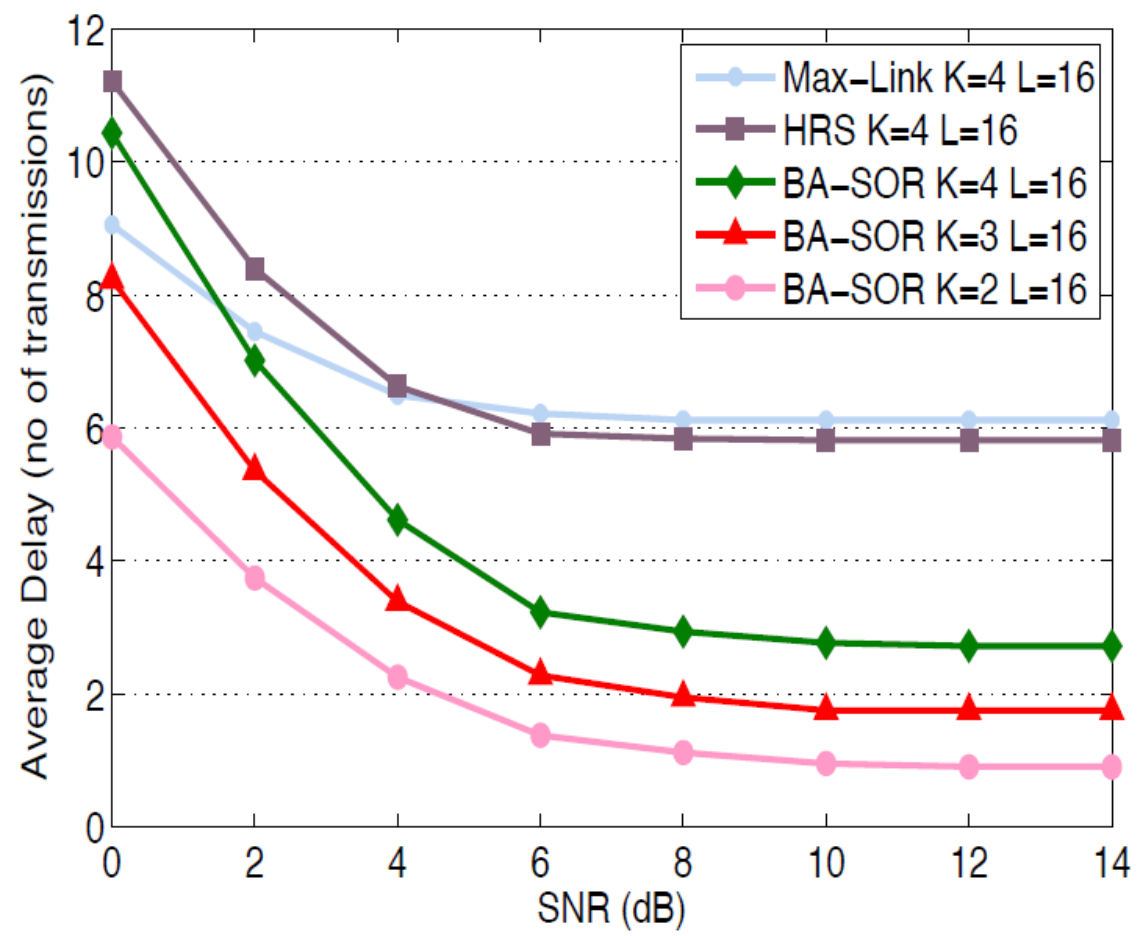

- The half-duplex schemes experience increased delay

- Due to successive transmissions packets tend to stay less in the buffers

- As relay number increases some packets tend to stay longer in the buffer as the probability to select each relay decreases 


\section{Conclusions}

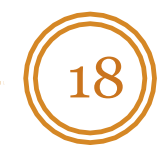

- In the proposed BA-SOR scheme in each timeslot, a relay-pair is selected and thus activated for transmission and reception

o Diversity is increased via opportunistic relay-pair selection

- The half-duplex loss of relaying is leveraged through successive transmissions

- The outage probability for small buffer sizes is worst than the half-duplex schemes

o IRI is introduced in the network

- Two possible interference mitigation techniques

- Interference cancellation at the receiving relay

- Interference avoidance when cancellation is not feasible

- Numerical results indicate that BA-SOR leverages the disadvantages of half-duplex relaying in terms of average capacity and average delay at the cost of increased outage probability 


\section{Future directions}

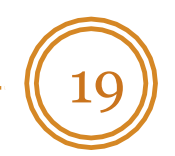

- Future directions include:

- The combination of BA-SOR with a more robust half-duplex scheme in order to merge the best of both techniques

o The investigation of additional important network parameters such as power consumption

- More efficient ways of interference mitigation and exploitation can be examined based on network coding aiming to increase the diversity of the network

o The development of distributed coordination aiming to reduce the CSI overhead 


\section{References}

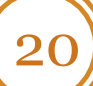

[Laneman et al., 2004] J. N. Laneman, D. N. C. Tse, and G. W. Wornell, "Cooperative diversity in wireless networks: Efficient protocols and outage behavior”, IEEE Trans. Inform. Theory, vol. 50, pp. 3062-3080, Dec. 2004.

- $\quad$ [Bletsas et al., 2006] A. Bletsas, A. Khisti, D. Reed, and A. Lippman, "A simple cooperative diversity method based on network path selection,” IEEE J. Select. Areas Commun., vol. 24, pp. 659-672, March 2006.

- $\quad$ [Ding et al., 2012] Z. Ding, I. Krikidis, B. Rong, J. S. Thompson, C. Wang, and S. Yang, "On combating the half-duplex constraint in modern cooperative networks: protocols and techniques,” IEEE Wireless Commun., vol. 19, pp. 20-27, Dec. 2012.

[Michalopoulos et al., 2008] D. S. Michalopoulos and G. K. Karagiannidis, "Performance analysis of single relay selection in Rayleigh fading," IEEE Trans. Wireless Commun., vol. 7, pp. 3718-3724, Oct. 2008.

[Krikidis et al., 2009] I. Krikidis, J. S. Thompson, Steve McLaughlin and Norbert Goertz, "Max-min relay selection for legacy amplify-and-forward systems with interference," IEEE Trans Wireless Commun., vol. 8, pp. 3016-3027, June 2009.

- $\quad$ [Nomikos et al., 2012/1] N. Nomikos and D. Vouyioukas, "A successive opportunistic relaying protocol with inter-relay interference mitigation,” in Proc. IEEE Wireless Commun. Mob. Comp. Conf, Limassol, Cyprus, Aug. 2012 , pp. 1-5.

- [Nomikos et al., 2012/2] N. Nomikos, D. N. Skoutas, D. Vouyioukas, C. Verikoukis, and C. Skianis, "Capacity maximization through energy-aware multi-mode relaying", Springer Wireless Pers. Commun. DOI 10.1007/ s11277-012-0899-5, 2012.

- $\quad$ [Ikhlef et al., 2012] A. Ikhlef, D. S. Michalopoulos, and R. Schober, "Max-max relay selection for relays with buffers," IEEE Trans. Wireless Commun., vol. 11, pp. 1124-1135, March 2012.

- $\quad$ [Krikidis et al., 2012] I. Krikidis, T. Charalambous, and J. S. Thompson, "Buffer-aided relay selection for cooperative diversity systems without delay constraints, “ IEEE Trans. Wireless Commun., vol. 11, pp. 1957-1967, May 2012.

- $\quad$ [Ikhlef et al., 2012/2] A. Ikhlef, K. Junsu, and R. Schober, "Mimicking full-duplex relaying using half-duplex relays with buffers," IEEE Trans. Vehicular Tech., vol. 61, pp. 3025-3037, Sept. 2012. 